\title{
Validation d'une technique de dosage multi- élémentaire des métaux par ICP-MS dans les milieux biologiques**
}

\section{Multielementary ICP-MS validation of metals determination in biological fluids $* *$}

Jean-Pierre GOULLÉ ${ }^{(1) *}$, Loïc MAHIEU ${ }^{(1)}$, Julien CASTERMANT ${ }^{(1)}$, Nicolas NEVEU(1), Gilbert LAINE ${ }^{(1)}$, Marie-Pascale NOUVEAU ${ }^{(1)}$, Roseline GEHANNE ${ }^{(2)}$, Daniel BOUIGE ${ }^{(3)}$, Christian LACROIX ${ }^{(1)}$

(1) Laboratoire de Pharmacocinétique et de Toxicologie Cliniques, Groupe Hospitalier du Havre BP 24 - 76083 LE HAVRE CEDEX

(2) Service de Médecine Préventive, Groupe Hospitalier du Havre - BP 24 - 76083 LE HAVRE CEDEX

(3) Laboratoire de Biochimie, Groupe Hospitalier du Havre - BP 24 - 76083 LE HAVRE CEDEX

*Auteur à qui adresser la correspondance : Jean-Pierre GOULLÉ, Laboratoire de Pharmacocinétique et de Toxicologie Cliniques, Groupe Hospitalier du Havre - BP 24 - 76083 LE HAVRE CEDEX Tél : 0232733223 - Fax : 0232733238 -E-mail : jgoulle@ch-havre.fr

** Ce travail a été présenté au XI $\mathrm{XI}^{\mathrm{ene}}$ congrès de la Société Française de Toxicologie Analytique, 11-13 juin 2003, DINARD - FRANCE 


\section{RÉSUMÉ}

Les auteurs présentent la validation d'une technique de dosage multi-élémentaire des métaux dans le sang total, le plasma et les urines par plasma à couplage inductif relié à un détecteur de masse (ICP-MS). Pour pouvoir être utilisée en routine, celle-ci doit permettre la détermination d'un grand nombre d'éléments à partir d'une préparation unique pour chaque matrice. Les mesures sont réalisées sur un spectromètre X7 CCT Thermo Elemental, sans cellule dynamique de réaction, équipé d'un passeur d'échantillon et du logiciel PlasmaLab. Les métaux suivants sont quantifiés simultanément dans les échantillons biologiques par dilution en milieu acide en présence de triton : $\mathrm{Li}, \mathrm{Be}, \mathrm{B}, \mathrm{Al}, \mathrm{Mn}, \mathrm{Co}, \mathrm{Ni}, \mathrm{Cu}$, $\mathrm{Zn}, \mathrm{Ga}, \mathrm{Ge}, A s, \mathrm{Se}, \mathrm{Rb}, \mathrm{Sr}, \mathrm{Mo}, \mathrm{Pd}, \mathrm{Cd}, \mathrm{Sn}, \mathrm{Sb}, \mathrm{Te}, \mathrm{Ba}, \mathrm{W}, \mathrm{Pt}$, $\mathrm{Hg}, \mathrm{Tl}, \mathrm{Pb}, \mathrm{Bi}, \mathrm{U}$. Le protocole de validation utilisé est celui recommandé par Société Française de Biologie Clinique $(S F B C)$. Pour les urines, les résultats sont rapportés à la créatinine, dont le dosage est réalisé par technique enzymatique. Tous les éléments sont validés soit dans les trois milieux biologiques (sang total, plasma, urines), soit dans deux milieux, soit dans un. Les coefficients de régression sont dans la gamme de linéarité (de 0 à 25 ou $1000 \mu \mathrm{g} / \mathrm{l}$ selon les éléments) supérieurs à 0,999. Les limites de quantification s'échelonnement de $0,003 \mu \mathrm{g} / \mathrm{l}(\mathrm{U})$ à $10 \mu \mathrm{g} / \mathrm{l}(\mathrm{B})$ pour le sang total, de $0,003 \mu \mathrm{g} / \mathrm{l}(U)$ à $17 \mu \mathrm{g} / \mathrm{l}$ (Al) pour le plasma et de $0,002 \mu \mathrm{g} / \mathrm{l}(U)$ à $17 \mu \mathrm{g} / \mathrm{l}$ (Se) pour les urines. L'évolution rapide et récente de I'ICP-MS constitue un apport déterminant en biologie clinique et plus particulièrement dans le domaine de l'analyse toxicologique en raison de la puissance de l'outil, offrant des perspectives nouvelles pour une meilleure prise en charge des malades.

\section{MOTS-CLES}

Plasma à couplage inductif, détecteur de masse, métaux, métalloïdes.

\section{Introduction}

La recherche et le dosage des métaux dans les milieux biologiques dans le cadre de déterminations multi-élémentaires se heurtent encore au plan de la réalisation technique à des difficultés importantes. Les méthodes de routine font appel à la spectrophotométrie d'absorption atomique (SAA) dans ses deux modes : SAA avec flamme et SAA électrothermique. Si la première peut être facilement mise en œuvre pour traiter de grandes séries, pour l'immense majorité des métaux, sa sensibilité n'est pas suffisante. Le nombre de métaux que l'on peut doser par cette méthode est de l'ordre de vingt. Quant à la seconde, si elle dispose d'une sensibilité supérieure permettant de quantifier environ quarante minéraux, elle est encore insuffisante pour mesurer certains éléments comme le bore, le brome, l'étain ou l'iode, par exemple. La SAA est essentiellement conçue et utilisée pour réaliser des dosages monoélémentaires et sa cadence analytique est peu adaptée aux grandes séries, en particulier dans le mode le plus sensible de SAA électrothermique qui nécessite souvent l'emploi d'ajouts dosés sur chaque échantillon, ce qui

\section{SUMMARY}

Three multi-elementary quantitation methods using inductively coupled plasma mass spectrometry (ICP-MS) are developed and validated in human serum, in whole blood and in urine. In order to be routinely used, the latter must allow the simultaneous determination of several elements, using a single sample preparation procedure. The ICP-MS measurements were done using a Thermo Elemental $X 7$ series and PlasmaLab software without dynamic reaction cell. Deionised purified water was prepared extemporaneously. Many elements are simultaneously quantified in biological fluids : $L i, B e, B, A l, M n, C o, N i, C u, Z n, G a, G e, A s, S e, R b$, $\mathrm{Sr}, \mathrm{Mo}, \mathrm{Pd}, \mathrm{Cd}, \mathrm{Sn}, \mathrm{Sb}, \mathrm{Te}, \mathrm{Ba}, \mathrm{W}, \mathrm{Pt}, \mathrm{Hg}, \mathrm{Tl}, \mathrm{Pb}, \mathrm{Bi}, \mathrm{U}$. For urines, the results are corrected after enzymatic ceratinine determination. To be validated, each element has to force : linearity with correlation coefficient higher than 0.99 . Repeatability and reproducibility variation coefficient are respectively better than $5 \%$ and $10 \%$. All the elements have been validated either in one, two, or the three matrixes, from 0 to 25 or $1000 \mu \mathrm{g} / \mathrm{l}$ owing to the element. Quantitation limits range from $0.003 \mu \mathrm{g} / \mathrm{l}(U)$ to $10 \mu \mathrm{g} / \mathrm{l}(\mathrm{B})$ for the whole blood, from $0.003 \mu \mathrm{g} / \mathrm{l}(\mathrm{U})$ to $17 \mu \mathrm{g} / \mathrm{l}$ (Al) for the plasma and from $0.002 \mu \mathrm{g} / \mathrm{l}(U)$ to $17 \mu \mathrm{g} / \mathrm{l}$ (Se) for urines. ICP-MS major advances in the field of clinical biology are very important especially for toxicological analysis as this powerfull equipment allows better patient take in care.

\section{KEY-WORDS}

Inductively coupled plasma, mass detector, metals, metalloids.

limite considérablement la cadence analytique. Enfin le dosage dans les milieux biologiques d'un certain nombre d'éléments réfractaires, les lanthanides ou les actinides, n'est accessible que par la torche à plasma même si l'intérêt de la plupart d'entre eux en biologie est limité. L'introduction de la torche à plasma (ICP ou Inductively Coupled Plasma), les progrès analytiques relativement récents grâce au détecteur de masse (ICPMS), mais également les avancées dans le domaine du traitement des signaux et du pilotage des appareils, améliorent considérablement cette approche et ouvrent des perspectives nouvelles pour l'exploration des métaux tant en pratique clinique qu'en médecine légale ou que sur l'impact des effets de l'environnement chez l'homme $(1,2)$. L'apparition très récente sur le marché d'ICP-MS compacts dits de «paillasse» avec des logiciels de pilotage élaborés et conviviaux met à la disposition des laboratoires des appareils d'une nouvelle génération offrant la possibilité de mesurer simultanément un nombre important d'éléments à partir d'un échantillon unique (sang, plasma, urines, cheveux), avec une cadence analytique élevée. Grâce au détecteur 
de masse, les performances de la torche à plasma sont du plus haut niveau, cette technique étant caractérisée par d'excellentes précision et exactitude, ainsi qu'une bonne spécificité pour la plupart des éléments. Le niveau de sensibilité moyen est supérieur à la SAA électrothermique (figure 1). En termes de performances pures l'ICP-MS permet d'atteindre des niveaux de sensibilité jamais égalés par les autres techniques (tableau I). Le principe de la mesure repose sur le fait que l'échantillon amené jusqu'à la torche à plasma par une pompe péristaltique est nébulisé puis atomisé à très haute température dans un plasma. Le plasma est généré par le couplage inductif d'électrons libres avec des oscillations rapides du champ électromagnétique à la fréquence de $27 \mathrm{Mhz}$ dans l'argon. Ce gaz présente de nombreux avantages : il est relativement inerte chimiquement et s'obtient facilement avec un haut degré de pureté, limitant de ce fait les contaminations éventuelles. Au contact de l'argon, l'échantillon est nébulisé puis transporté jusqu'au centre du plasma où la température est proche de $8000 \mathrm{~K}$. Il est alors atomisé puis ionisé sous forme de cations monovalents. Dans ces conditions, les éléments métalliques, les métalloïdes, les alcalins, les alcalino-terreux, les lanthanides et les actinides sont ionisés à plus de $90 \%$. Le carbone, l'azote, l'oxygène, le soufre, le mercure et les halogènes ne sont que partiellement ionisés. Cette ionisation partielle est malgré tout suffisante pour quantifier le brome et l'iode dans les milieux biologiques. Malheureusement à la faveur de ces températures élevées, des combinaisons peuvent se produire par exemple entre l'argon de masse 40 et des atomes de la matrice biologique, le chlore de masse 35 ou/et le carbone de masse 12 pour donner des molécules de masse respective 75 et 52 rendant tout à fait aléatoire les mesures d'As et de $\mathrm{Cr}$ dont les masses atomiques sont 75 et 52 . Pour l'As, l'interférence à la masse 75 avec $\mathrm{ArCl}$ peut être contournée dans les appareils disposant dans leur logiciel d'un algorithme de calcul permettant de tenir compte de la quantité de chlore présente dans la matrice biologique et donc de la quantité d' $\mathrm{ArCl}$ formée qui est déduite. En ce qui concerne le chrome, l'interférence à la masse 52 due à la présence d'ArCl nécessite l'emploi d'une cellule dynamique de réaction (DRC) ou d'un ICP-MS à haute résolution qui mesure la masse avec une plus grande précision. Dans l'appareil d'ICP-MS, une interface composée de deux cônes de nickel et d'une série de lentilles permet de stopper les photons et de focaliser les ions pour les amener au quadripôle où ils sont séparés en fonction du rapport de leur masse atomique sur leur charge. Un détecteur permet de quantifier l'intensité du signal pour chaque masse (photographies 1 et 2). L'ensemble du système est piloté et suivi par une station informatique. Les techniques d'analyse en biologie de quelques métaux et métalloïdes, ainsi que l'interprétation des valeurs obtenues ont été éditées dans deux ouvrages français $(3,4)$. Depuis quelques années, le Journal of Analytical Atomic Spectrometry publie chaque année une synthèse bibliographique des articles parus dans ce domaine et en particulier par ICP-MS (5-11). L'objectif principal de ce travail est de valider une technique multiélémentaire du dosage simultané d'un grand nombre d'éléments dans trois matrices biologiques (sang total, plasma, urines). Compte tenu de la très grande sensibilité de la technique, nous avons cherché à optimiser un mode de préparation, si possible unique pour chaque matrice, permettant de quantifier simultanément le plus grand nombre d'éléments. Cette étape préliminaire est nécessaire en vue de la détermination des valeurs habituelles dans une population témoin, préalable à l'utilisation d'un dépistage multiélémentaire dans le cadre de la toxicologie clinique ou médico-légale ou de programmes hospitaliers de recherche clinique axés sur les métaux et consacrés à des pathologies variées.

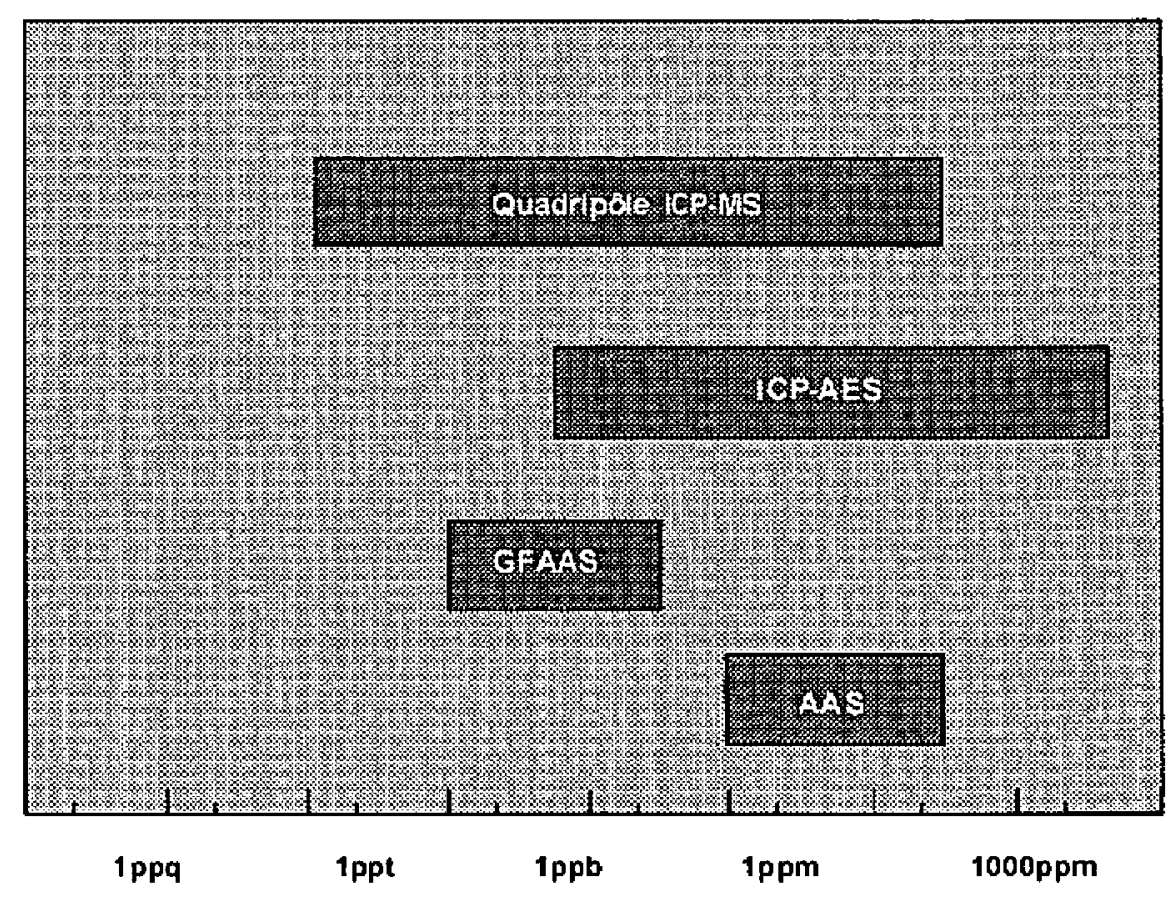

Figure 1 : Sensibilités moyennes comparées de l'absorption atomique et de la torche à plasma.

Tableau I : Les performances de l'ICP-MS.

\begin{tabular}{|l|l|}
\hline \multicolumn{1}{|c|}{ Ppt ou ng/ } & \multicolumn{1}{c|}{ Éléments } \\
\hline $0,1-1,0$ & $\mathrm{Li}, \mathrm{Be}, \mathrm{Mg}, \mathrm{V}, \mathrm{Cr}, \mathrm{Mn}, \mathrm{Co}, \mathrm{Ga}, \mathrm{Rb}, \mathrm{Sr}, \mathrm{Y}, \mathrm{Nb}, \mathrm{Mo}, \mathrm{Tc}$, \\
& $\begin{array}{l}\mathrm{Ru}, \mathrm{Rh}, \mathrm{Pd}, \mathrm{Ag}, \mathrm{Cd}, \mathrm{In}, \mathrm{Sb}, \mathrm{Cs}, \mathrm{Ba} \mathrm{La}, \mathrm{Ta}, \mathrm{Re}, \mathrm{Pt}, \mathrm{Hg}, \\
\mathrm{Tl}, \mathrm{Bi}, \mathrm{Fr}, \mathrm{Ra}, \mathrm{Ac}, \mathrm{Ce}, \mathrm{Pr}, \mathrm{Nd}, \mathrm{Pm}, \mathrm{Sm}, \mathrm{Eu}, \mathrm{Gd}, \mathrm{Tb}, \mathrm{Dy}, \\
\mathrm{Ho}, \mathrm{Er}, \mathrm{Tm}, \mathrm{Yb}, \mathrm{Lu}, \mathrm{Th}, \mathrm{Pa}, \mathrm{U}, \mathrm{Np}, \mathrm{Pu}, \mathrm{Am}\end{array}$ \\
\hline $1,0-10$ & $\begin{array}{l}\mathrm{Al}, \mathrm{Sc}, \mathrm{Ti}, \mathrm{Ni}, \mathrm{Cu}, \mathrm{Zn}, \mathrm{Ge}, \mathrm{As}, \mathrm{Zr}, \mathrm{Sn}, \mathrm{Te}, \mathrm{Hf}, \mathrm{W}, \mathrm{Ir}, \\
\mathrm{Au}, \mathrm{Pb}\end{array}$ \\
\hline $10-100$ & $\mathrm{~B}, \mathrm{Na}, \mathrm{Se}, \mathrm{Br}, \mathrm{I}, \mathrm{Os}$ \\
\hline $100-10 \mu \mathrm{g} / \mathrm{l}(\mathrm{ppb})$ & $\mathrm{P}, \mathrm{Cl}, \mathrm{K}, \mathrm{Ca}, \mathrm{Fe}$ \\
\hline$>10 \mu \mathrm{g} / \mathrm{l}$ & $\mathrm{Si}, \mathrm{S}$ \\
\hline
\end{tabular}




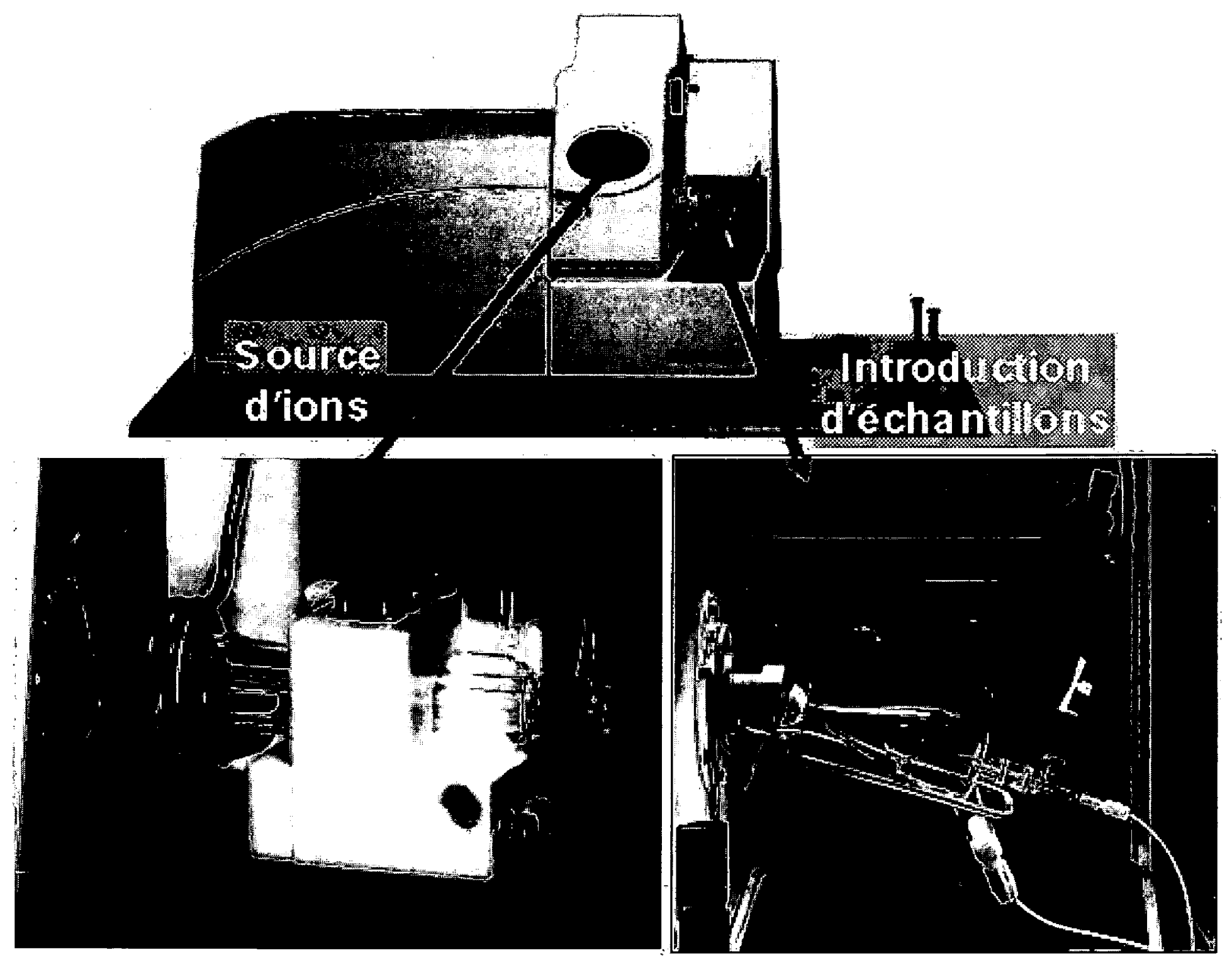

Photographie 1 : vue éclatée d'un ICP-MS (I).

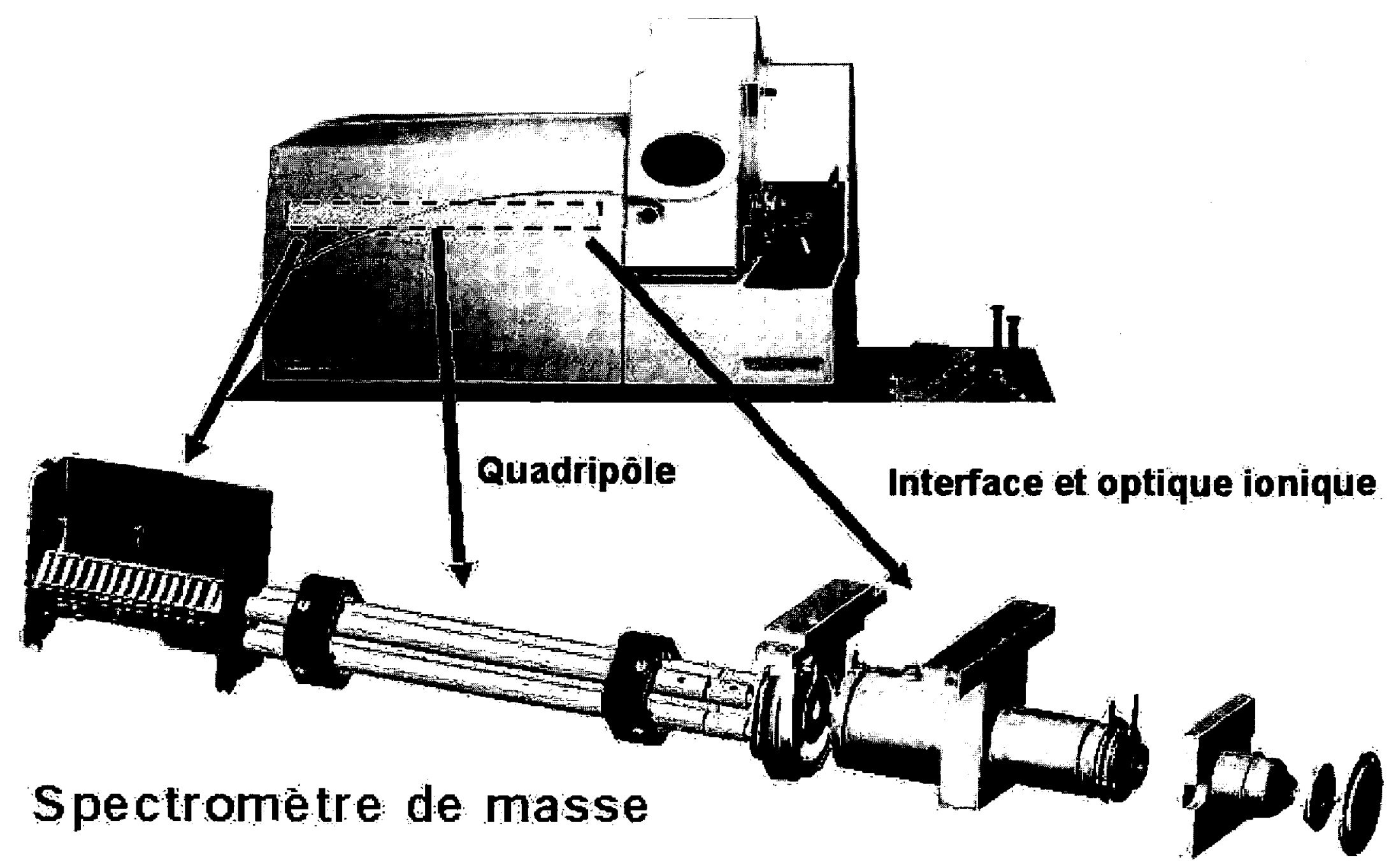

Photographie 2 : vue éclatée d'un ICP-MS (2). 


\section{Matériel et méthodes}

\section{Instrumentation :}

Il s'agit d'une torche à plasma de type ThermoOptek X Series couplée à un spectromètre de masse, modèle X7/CCT (ThermoOptek, Courtaboeuf, France).

L'appareil est équipé d'une torche en quartz de $1,5 \mathrm{~mm}$, d'un nébuliseur concentrique en verre borosilicaté de $1 \mathrm{ml}$ (type concentrique de Marque Glass Expansion référence ThermoElemental 1201318) avec un débit d'échantillon de $0,85 \mathrm{ml} / \mathrm{min}$, d'une chambre de nébulisation en quartz munie d'un refroidisseur à effet Peltier régulant sa température à $3^{\circ} \mathrm{C}$, et d'un passeur d'échantillons de type CETAC ASX-510. L'ensemble des données est enregistré sur une station informatique dotée du logiciel d'analyse PlasmaLab version 2.0 sous Windows NT. Les paramètres instrumentaux sont indiqués tableau II. Le dosage de la créatinine urinaire est réalisé par technique enzymatique sur Intégra 400 (Roche, Meylan, France).

Tableau II : Paramètres instrumentaux utilisés pour le dosage muti-élémentaire.

\begin{tabular}{|l|lr|}
\hline \multicolumn{2}{|l}{ Puissance induite (torche) } & $1200 \mathrm{~W}$ \\
\hline \multirow{3}{*}{ Débits d'argon } & Plasmagène & $15 \mathrm{~L} / \mathrm{min}$ \\
& Nébuliseur & $0,95 \mathrm{~L} / \mathrm{min}$ \\
& Auxiliaire & $0,66 \mathrm{~L} / \mathrm{min}$ \\
\hline \multirow{2}{*}{ Interface } & Cône échantillonneur (nickel) & orifice $: \varnothing 1 \mathrm{~mm}$ \\
& Cône écorceur (nickel) & orifice : $\varnothing 0,4 \mathrm{~mm}$ \\
\hline \multirow{2}{*}{ Vide } & Interface & $1,9 \mathrm{mbar}$ \\
& Quadripole & $1,6.10^{-7} \mathrm{mbar}$ \\
\hline
\end{tabular}

\section{Réactifs :}

La source de plasma utilisée est de l'argon présentant un haut degré de pureté, supérieur à $99,999 \%$ (Linde gas, Gargenville, France). L'eau utilisée est purifiée extemporanément sur Milli-QPLUS 185 (Millipore, St Quentin en Yvelines, France). L'acide nitrique à $65 \%$ de qualité suprapur, le triton X100 et les solutions multi-élémentaires (multiélément standard solution VI contenant 30 éléments à $10 \mathrm{ou} 100 \mathrm{mg} / \mathrm{l}$ ) proviennent de chez Merck (Darmstadt, Allemagne). Des étalons individuels de W, Pt, Pd, Hg, Sb, Sn, Ge (CPI international, Amsterdam, Hollande) sont ajoutés à ces solutions multi-élémentaires. L'étalonnage interne de l'appareil est réalisé avec une solution de Rhodium (CPI international). La validité des dosages est vérifiée pour le plasma, le sang total et les urines par des contrôles titrés par ICP-MS Seronorm niveau 1 (Trace elements serum level 1 - réf. 201405 ; Trace elements whole blood 2 - réf. 201607 ; Trace elements urine - réf.
201207 ; SERO AS, Billingstade, Norvège). Le sang est prélevé dans un tube sous vide de $7 \mathrm{ml}$ hépariné pour éléments traces de type "Vacutainer ${ }^{\circledR}$ 》 référence 367735 (Beckton Dickinson, Le Pont de Claix, France). La moitié de chaque prélèvement sanguin est décantée dans un tube en polystyrène de $5 \mathrm{ml}$ puis centrifugée dans un délai de 2 heures après le prélèvement et le plasma immédiatement décanté. Le sang total, le plasma décanté sont congelés avant analyse. Les urines sont recueillies sur un flacon de $30 \mathrm{ml}$ en polystyrène transparent muni d'un bouchon à vis en polypropylène et également congelées avant analyse. Le laboratoire participe au contrôle qualité ICP-MS canadien (Programme de comparaisons interlaboratoires, Sainte Foy, Canada) qui adresse trois fois par an à ses adhérents du sang total, des urines et des cheveux.

\section{Préparation des gammes d'étalonnage :}

Les gammes d'étalonnage permettant le dosage multiélémentaire dans le plasma sont réalisées en solution aqueuse, directement dans la solution diluante. En fait, l'étalonnage se fait par l'intermédiaire d'une gamme de concentrations en 8 points, préparée dans une solution d'acide nitrique à $0,144 \mathrm{M}$, s'échelonnant de 0 à $24 \mu \mathrm{g} / \mathrm{l}$ $(0-0,5-1-2-3-6-12$ et $24 \mu \mathrm{g} / \mathrm{l})$ ou de 0 à $240 \mu \mathrm{g} / \mathrm{l}(0-5-$ 10-20-30-60-120 et $240 \mu \mathrm{g} / \mathrm{l}$ ) selon l'élément. En ce qui concerne le sang total, le dosage multi-élémentaire est effectué par la méthode des ajouts dosés, autrement dit par une gamme de concentrations préparée à l'aide d'un pool de sang, dans la matrice considérée. Une gamme d'ajouts dosés est réalisée, s'échelonnant de $X$ à $X+24 \mu \mathrm{g} / 1(\mathrm{X}, \mathrm{X}+0,5, \mathrm{X}+1, \mathrm{X}+3, \mathrm{X}+6, \mathrm{X}+12$ et $\mathrm{X}+24$ $\mu \mathrm{g} / \mathrm{l})$ ou de $X$ à $X+240 \mu \mathrm{g} / \mathrm{l}(\mathrm{X}, \mathrm{X}+5, \mathrm{X}+10, X+30$, $X+60, X+120$ et $X+240 \mu \mathrm{g} / \mathrm{l})$ selon les éléments. Pour les urines deux dilutions différentes sont utilisées en fonction de la nature de l'élément :

- au dixième, dans ce cas il s'agit de la même gamme que pour le plasma,

- au cinquième avec des ajouts dosés aux mêmes surcharges dans la matrice à l'aide d'un pool d'urines, que pour le sang total.

\section{Préparation des échantillons :}

Les échantillons plasmatiques sont préparés par dilution au dixième, dans une solution aqueuse composée d'acide nitrique à $0,144 \mathrm{M}$ et de triton à $0,01 \%(\mathrm{v} / \mathrm{v})$, dans des tubes en polypropylène. Les échantillons de sang, préalablement congelés à $-20^{\circ} \mathrm{C}$ puis décongelés, sont dilués au dixième dans une solution aqueuse composée d'acide nitrique à $0,144 \mathrm{M}$ et de triton à $0,1 \%$ $(\mathrm{v} / \mathrm{v})$ dans des tubes en polypropylène. Une fois l'hémolyse achevée, les mélanges sont centrifugés à 4000 tours/min. Les urines sont préparées par dilution au 1 : 10 et au $1: 5$ dans l'acide nitrique $0,216 \mathrm{M}$ et de triton 
à $0,01 \%$ v/v en fonction de l'élément à doser. Pour des raisons pratiques, les mesures sont réalisées sur une miction, les résultats étant rapportés à la créatinine déterminée par voie enzymatique. La quantité d'échantillon sanguin, plasmatique ou urinaire nécessaire est de $0,4 \mathrm{ml}$.

\section{Conditions de validation :}

Le protocole de validation utilisé pour ces méthodes est celui recommandé par la Société Française de Biologie Clinique (SFBC) (12). Un pool est préparé pour la validation du dosage des métaux dans le plasma et les urines, alors que pour le sang total, il est fait appel au contrôle Seronorm. Pour être validé, le dosage d'un élément doit répondre à des critères de validation établis.

Avant de réaliser ces dosages multi-élémentaires dans les trois matrices, différentes solutions de dilution ont été essayées avec des étalons titrés par ICP-MS pour optimiser les concentrations en acide nitrique et en triton. Pour évaluer l'influence d'un éventuel effet de matrice dans le plasma, des gammes de concentration ont été réalisées : l'une en solution aqueuse, l'autre dans la matrice plasma. Les résultats obtenus étant superposables, l'étalonnage est réalisé en milieu aqueux pour l'ensemble des dosages effectués dans le plasma. En ce qui concerne le sang total, en raison d'un effet de matrice non négligeable pour certains éléments, l'étalonnage est réalisé selon la méthode des ajouts dosés en utilisant un pool de sang. Pour chaque élément, la courbe de calibration est établie à l'aide de 8 niveaux différents de concentrations, de 0 à $24 \mu \mathrm{g} / \mathrm{l}$ ou de 0 à $240 \mu \mathrm{g} / \mathrm{l}$ en cas d'étalonnage aqueux; de X à $\mathrm{X}+24 \mu \mathrm{g} / \mathrm{l}$ ou de $\mathrm{X}$ à $\mathrm{X}+240 \mu \mathrm{g} / \mathrm{l}$ en cas d'étalonnage selon la méthode des ajouts dosés. Chaque mesure est réalisée en triple. La linéarité est validée pour chaque élément par l'établissement de la droite de régression sur la plage de concentrations considérée ainsi que par le calcul du coefficient de corrélation. La récupération d'ajouts est également mesurée dans les trois matrices : sang total, plasma, urines. L'exactitude est appréciée par deux types de contrôles : un contrôle intralaboratoire constitué par des étalons titrés par ICPMS (Seronorm) pour chacune des matrices ; un contrôle interlaboratoires pour ICP-MS pour le sang total et les urines (contrôle canadien). Pour chaque élément, les limites de détection et de quantification sont calculées selon les recommandations de la SFBC. En ce qui concerne la limite de détection (LD), celle-ci est définie comme la concentration minimale pour laquelle la réponse peut être distinguée, avec une certaine probabilité du blanc. Elle est estimée, en réalisant des mesures répétées de ce blanc $(n=30)$, par : $L D(p p b)=$ $\left(\mathrm{vm}_{\mathrm{bl}}+3 \mathrm{ET}\right) \div \mathrm{a}$. La limite de quantification (LQ) quand à elle correspond à la concentration minimale quantifiable avec une précision acceptable et pouvant être distinguée du blanc. Elle est obtenue, par la même série de mesures que pour la limite de détection, par : LQ $(\mathrm{ppb})=\left(\mathrm{vm}_{\mathrm{bl}}+10 \mathrm{ET}\right) \div \mathrm{a}$.

\section{Résultats : Dosage multi-élémentaire des métaux dans le plasma, le sang total et}

\section{les urines}

L'analyse de chaque mélange préparé (plasma, sang total, urines) permet de calculer pour tous les éléments l'équation de la droite de régression ainsi que le coefficient de régression de chaque courbe d'étalonnage. Compte tenu du grand nombre d'éléments quantifiés et des dosages réalisés dans trois matrices différentes, soit 80 droites de régression, seule la pente de la droite de régression est reportée dans les tableaux III pour le plasma, IV pour le sang total, $\mathrm{V}$ pour les urines. Les limites de détection et de quantification peuvent également être consultées dans les tableaux II, IV, V. Pour ce qui est de la répétabilité et de la reproductibilité, les concentrations auxquelles elles ont été mesurées sont indiquées dans les trois tableaux.

\section{Discussion}

Le but de ce travail a été de valider dans le cadre d'une étude multi-élémentaire simultanée, l'analyse du plus grand nombre possible d'éléments. Cependant, un certain nombre de considérations et de contraintes ont limité cette approche. La torche à plasma couplée à un détecteur de masse n'est capable de détecter que les isotopes de 78 éléments parmi les 110 éléments de la table périodique excluant par exemple le carbone, le fluor, le chlore ou encore la majorité des actinides. Par ailleurs, un certain nombre d'éléments comme les alcalins ou les alcalino-terreux (sodium, potassium, calcium, magnésium) ou le fer ont été exclus compte tenu du fait qu'il existe de nombreuses méthodes automatisées parfaitement adaptées à leur analyse en routine et de leur faible intérêt dans un cadre toxicologique. Enfin, le dosage du chrome de masse 52 n'a pas été réalisé en raison de l'existence d'une interférence polyatomique due à la combinaison à la haute température de la torche entre l'argon (de masse 40) et le carbone (de masse 12). Le dosage du vanadium n'a pu être réalisé en raison d'interférences. Pour lever ces interférences, l'emploi d'une cellule dynamique de réaction est indispensable (DRC).

Les résultats montrent que la validation de cette technique a pu être réalisée en simultané pour 27 éléments dans le plasma, 24 dans le sang total et pour 29 élé- 
Tableau III : Validation du dosage multi-élémentaire des métaux dans le plasma.

\begin{tabular}{|c|c|c|c|c|c|c|}
\hline $\begin{array}{l}\text { Élément } \\
\text { isotope }\end{array}$ & $\mathbf{r}$ & $\begin{array}{c}\mathrm{LD} \\
\mu \mathrm{g} / \mathrm{I}\end{array}$ & $\begin{array}{l}\mathrm{LQ} \\
\mu \mathrm{g} /\end{array}$ & $\begin{array}{l}\text { Répét. } \\
\text { CV \% }\end{array}$ & $\begin{array}{l}\text { Repro. } \\
\text { CV \% }\end{array}$ & $\begin{array}{c}\text { Conc. } \\
\mu \mathrm{g} / \mathrm{l}\end{array}$ \\
\hline $7 \mathrm{Li}$ & 0,9999 & 0,44 & 0,89 & 1,03 & 5,71 & 83,0 \\
\hline $9 \mathrm{Be}$ & 0,9999 & 0,13 & 0,20 & 0,85 & 5,20 & 50,3 \\
\hline $11 \mathrm{~B}$ & 0,9997 & 9,00 & 12,00 & 1,66 & 3,26 & 124 \\
\hline $27 \mathrm{AI}$ & 0,9991 & 12,00 & 17,40 & 1,25 & 13,76 & 9,9 \\
\hline $55 \mathrm{Mn}$ & 0,9999 & 0,37 & 0,42 & 0,60 & 2,59 & 7,7 \\
\hline $59 \mathrm{Co}$ & 0,9997 & 0,21 & 0,41 & 0,86 & 4,24 & 6,6 \\
\hline $60 \mathrm{Ni}$ & 0,9874 & 1,31 & 1,50 & 1,58 & 5,13 & 11,8 \\
\hline $65 \mathrm{Cu}$ & 0,9978 & 1,47 & 1,81 & 0,49 & 2,35 & 1092 \\
\hline $66 \mathrm{Zn}$ & 0,9982 & 5,31 & 6,79 & 0,27 & 2,36 & 557 \\
\hline $69 \mathrm{Ga}$ & 0,9992 & 0,29 & 0,65 & 0,74 & 3,57 & 10,5 \\
\hline $72 \mathrm{Ge}$ & 0,9996 & 0,23 & 0,29 & 0,89 & 3,22 & 9,0 \\
\hline $75 \mathrm{As}$ & 0,9997 & 0,23 & 0,32 & 0,22 & 3,44 & 67,9 \\
\hline $82 \mathrm{Se}$ & 0,9998 & 6,36 & 7,90 & 1,42 & 1,61 & 124 \\
\hline $85 \mathrm{Rb}$ & 0,9995 & 0,12 & 0,15 & 0,26 & 3,06 & 130 \\
\hline $88 \mathrm{Sr}$ & 0,9998 & 0,12 & 0,15 & 0,35 & 2,14 & 45,1 \\
\hline $95 \mathrm{Mo}$ & 0,9997 & 0,07 & 0,13 & 1,09 & 2,46 & 8,8 \\
\hline $111 \mathrm{Cd}$ & 0,9998 & 0,02 & 0,04 & 2,64 & 2,88 & 4,7 \\
\hline $118 \mathrm{Sn}$ & 0,9979 & 0,44 & 1,11 & 0,86 & 2,03 & 6,5 \\
\hline $121 \mathrm{Sb}$ & 0,9996 & 0,02 & 0,03 & 3,23 & 4,03 & 5,1 \\
\hline $125 \mathrm{Te}$ & 0,9999 & 0,02 & 0,05 & 1,18 & 2,35 & 4,8 \\
\hline $137 \mathrm{Ba}$ & 0,9998 & 3,85 & 8,71 & 0,75 & 2,91 & 95,0 \\
\hline $182 W$ & 0,9997 & 0,30 & 0,74 & 2,17 & 4,05 & 5,9 \\
\hline $195 \mathrm{Pt}$ & 0,9977 & 0,03 & 0,05 & 0,97 & 4,50 & 5,4 \\
\hline $205 \mathrm{Ti}$ & 0,9996 & 0,01 & 0,02 & 1,51 & 4,43 & 4,9 \\
\hline $208 \mathrm{~Pb}$ & 0,9991 & 0,28 & 0,35 & 1,96 & 6,70 & 5,0 \\
\hline $209 \mathrm{Bi}$ & 0,9994 & 0,01 & 0,02 & 1,88 & 5,19 & 4,3 \\
\hline $238 \mathrm{U}$ & 0,9997 & 0,001 & 0,003 & 2,28 & 5,70 & 5,4 \\
\hline
\end{tabular}

$\mathrm{r}=$ coefficient de corrélation, $\mathrm{LD}=$ limite de détection, $\mathrm{LQ}=$ limite de quantification, Répét. = répétabilité,

Repro. $=$ reproductibilité, Conc. $=$ niveau de concentration ayant servi aux mesures de répétabilité et de reproductibilité.

ments dans les urines. L'amélioration de la maîtrise de l'appareillage au cours des mois explique que pour certains éléments, la reproductibilité -mesurée sur une période de 3 mois- soit inférieure à la répétabilité (tableaux III à V). Il convient de souligner que les critères de validation retenus sont relativement sévères, conçus essentiellement pour des techniques monoparamétriques adaptées à la recherche de variations physiologiques. Notre démarche consiste à mettre en évidence l'augmentation d'un ou plusieurs éléments pouvant avoir une conséquence toxique pour l'homme. Il eut été de ce fait plus logique de réaliser cette validation pour chaque élément à une concentration voisine de la limite physiologique supérieure. Malheureusement, nous nous sommes heurtés au fait que pour de nombreux éléments, les concentrations normales et/ou toxiques ne sont pas connues. En ce qui concerne l'aluminium dans le plasma, malgré une reproductibilité à $13,8 \%$, ce paramètre a été validé car ce résultat a été obtenu à une concentration voisine de la limite de quantification. Parmi tous les éléments mesurés, l'aluminium est le seul pour lequel les performances sont inférieures à 
Tableau IV : Validation du dosage multi-élémentaire des métaux dans le sang total.

\begin{tabular}{|c|c|c|c|c|c|c|}
\hline $\begin{array}{l}\text { Élément } \\
\text { isotope }\end{array}$ & $\mathbf{r}$ & $\begin{array}{l}\mathrm{LD} \\
\mu \mathrm{g} / \mathrm{f}\end{array}$ & $\begin{array}{l}\mathrm{LQ} \\
\mu \mathrm{g} / \mathrm{h}\end{array}$ & $\begin{array}{l}\text { Répét. } \\
\text { CV \% }\end{array}$ & $\begin{array}{l}\text { Repro. } \\
\text { CV \% }\end{array}$ & $\begin{array}{c}\text { Conc. } \\
\mu \mathrm{g} / \mathrm{l}\end{array}$ \\
\hline $9 \mathrm{Be}$ & 0,9996 & 0,13 & 0,23 & 2,61 & 2,78 & 5,9 \\
\hline $11 \mathrm{~B}$ & 0,9995 & 7,09 & 10,19 & 3,55 & 5,18 & 74,4 \\
\hline $55 \mathrm{Mn}$ & 0,9999 & 0,45 & 0,51 & 2,22 & 3,33 & 11,0 \\
\hline $59 \mathrm{Co}$ & 0,9999 & 0,31 & 0,35 & 2,25 & 7,34 & 5,5 \\
\hline $60 \mathrm{Ni}$ & 0,9999 & 4,91 & 5,34 & 3,71 & 9,99 & 5,4 \\
\hline $69 \mathrm{Ga}$ & 0,9997 & 0,08 & 0,11 & 1,24 & 7,23 & 5,5 \\
\hline $72 \mathrm{Ge}$ & 0,9999 & 0,52 & 0,64 & 2,18 & 9,39 & 16,7 \\
\hline $75 \mathrm{As}$ & 0,9998 & 0,20 & 0,28 & 3,36 & 5,35 & 14,4 \\
\hline $82 \mathrm{Se}$ & 0,9996 & 5,06 & 6,20 & 1,70 & 2,39 & 137 \\
\hline $85 \mathrm{Rb}$ & 0,9994 & 0,32 & 0,36 & 1,65 & 7,00 & 1150 \\
\hline $88 \mathrm{Sr}$ & 0,9999 & 0,09 & 0,11 & 1,02 & 2,36 & 30,5 \\
\hline $105 \mathrm{Pd}$ & 0,9998 & 0,05 & 0,07 & 2,59 & 9,28 & 0,14 \\
\hline $111 \mathrm{Cd}$ & 0,9999 & 0,03 & 0,05 & 2,18 & 2,87 & 6,3 \\
\hline $118 \mathrm{Sn}$ & 0,9999 & 0,15 & 0,20 & 3,46 & 6,74 & 0,62 \\
\hline $121 \mathrm{Sb}$ & 0,9997 & 0,03 & 0,05 & 1,61 & 3,79 & 26,7 \\
\hline $125 \mathrm{Te}$ & 0,9999 & 0,04 & 0,08 & 3,02 & 5,83 & 0,25 \\
\hline $137 \mathrm{Ba}$ & 0,9998 & 0,75 & 0,88 & 1,38 & 5,45 & 83,6 \\
\hline $182 \mathrm{~W}$ & 0,9999 & 0,04 & 0,06 & 1,58 & 7,33 & 0,16 \\
\hline $195 \mathrm{Pt}$ & 0,9994 & 0,008 & 0,02 & 1,85 & 5,83 & 0,01 \\
\hline $202 \mathrm{Hg}$ & 0,9998 & 0,63 & 0,81 & 3,09 & 4,23 & 7,3 \\
\hline $205 \mathrm{Ti}$ & 0,9999 & 0,005 & 0,008 & 2,76 & 3,43 & 5,4 \\
\hline $208 \mathrm{~Pb}$ & 0,9998 & 0,31 & 0,36 & 2,92 & 4,77 & 417 \\
\hline $209 \mathrm{Bi}$ & 0,9997 & 0,005 & 0,01 & 3,00 & 2,62 & 5,4 \\
\hline $238 \mathrm{U}$ & 0,9999 & 0,002 & 0,003 & 1,89 & 3,59 & 0,21 \\
\hline
\end{tabular}

$\mathbf{r}=$ coefficient de corrélation, $\mathrm{LD}=$ limite de détection, $\mathrm{LQ}=$ limite de quantification, Répét. = répétabilité,

Repro. $=$ reproductibilité, Conc. $=$ niveau de concentration ayant servi aux mesures de répétabilité et de reproductibilité.

celles de la spectrophotométrie d'absorption atomique électrothermique, technique utilisée en routine dans notre laboratoire pour de nombreux métaux et métalloïdes. En raison d'un effet mémoire important conduisant dans nos conditions à une très mauvaise répétabilité (coefficient de variation à 0,46 ) le mercure plasmatique n'a pas pu être validé, le dosage du mercure sur sang total en revanche montre d'excellentes performances. Pour l'argent, nous avons rencontré des problèmes d'exactitude et de reproductibilité nécessitant vraisemblablement une mise au point spécifique pour cet élément. Avec le palladium ce sont à la fois des problèmes de répétabilité, de reproductibilité et d'exactitude qui n'ont pas permis de le valider dans le plasma. Pour le sang total, nous avons délibérément choisi de ne pas quantifier le lithium, l'aluminium, le cuivre, le zinc, ces métaux étant habituellement dosés dans le plasma (et pour certains dans les globules rouges). La mesure du cuivre et du zinc est possible en utilisant une gamme plus étendue. Une des caractéristiques de cette technique est d'avoir une gamme de linéarité beaucoup plus importante que dans la majorité des techniques utilisées en biologie clinique $\left(10^{8}\right.$ contre $10^{2}$ à $\left.10^{3}\right)$. En ce qui concerne les urines, hormis l'argent et le vanadium, tous les éléments ont été validés. Dans les trois milieux biologiques étudiés, les limites de quantification obtenues s'échelonnent pour le sang total de 0,003 $\mu \mathrm{g} / \mathrm{l}(\mathrm{U})$ à $10 \mu \mathrm{g} / \mathrm{l}(\mathrm{B})$; pour le plasma de $0,003 \mu \mathrm{g} / \mathrm{l}(\mathrm{U})$ à $17 \mu \mathrm{g} / 1(\mathrm{Al})$; pour les urines de $0,002 \mu \mathrm{g} / \mathrm{l}(\mathrm{U})$ à 17 $\mu \mathrm{g} / \mathrm{l}$ (Se). Excepté pour l'aluminium, les sensibilités 
Tableau $V$ : Validation du dosage multi-élémentaire des métaux dans les urines.

\begin{tabular}{|c|c|c|c|c|c|c|}
\hline $\begin{array}{l}\text { Élément } \\
\text { isotope }\end{array}$ & $\mathbf{r}$ & $\begin{array}{l}\mathrm{LD} \\
\mu \mathrm{g} / \mathrm{l}\end{array}$ & $\begin{array}{l}\mathrm{LQ} \\
\mu \mathrm{g} / \mathrm{l}\end{array}$ & $\begin{array}{l}\text { Répét. } \\
\text { CV \% }\end{array}$ & $\begin{array}{l}\text { Repro. } \\
\text { CV \% }\end{array}$ & $\begin{array}{c}\text { Conc. } \\
\mu \mathrm{g} / \mathrm{l}\end{array}$ \\
\hline $7 \mathrm{Li}$ & 0,9998 & 0,19 & 0,23 & 3,08 & 6,03 & 11,2 \\
\hline $9 \mathrm{Be}$ & 0,9998 & 0,05 & 0,08 & 3,74 & 7,44 & 5,0 \\
\hline $11 \mathrm{~B}$ & 0,9997 & 24,90 & 31,70 & 4,47 & 3,42 & 983 \\
\hline $27 \mathrm{AI}$ & 0,9996 & 5,81 & 6,45 & 2,02 & 7,14 & 132 \\
\hline $55 \mathrm{Mn}$ & 0,9999 & 0,22 & 0,25 & 1,22 & 5,14 & 13,0 \\
\hline $59 \mathrm{Co}$ & 0,9999 & 0,22 & 0,26 & 1,15 & 2,87 & 10,0 \\
\hline $60 \mathrm{Ni}$ & 0,9999 & 1,26 & 1,42 & 1,59 & 3,50 & 40,0 \\
\hline $65 \mathrm{Cu}$ & 0,9999 & 0,68 & 0,90 & 1,40 & 3,35 & 28,0 \\
\hline $66 \mathrm{Zn}$ & 0,9999 & 2,98 & 3,91 & 1,09 & 8,25 & 450 \\
\hline $69 \mathrm{Ga}$ & 0,9999 & 0,01 & 0,02 & 2,02 & 3,88 & 2,39 \\
\hline $72 \mathrm{Ge}$ & 0,9994 & 0,16 & 0,20 & 2,40 & 8,87 & 3,90 \\
\hline $75 \mathrm{As}$ & 0,9999 & 0,24 & 0,39 & 0,72 & 3,91 & 100 \\
\hline $82 \mathrm{Se}$ & 0,9995 & 14,80 & 16,90 & 3,55 & 5,52 & 31,6 \\
\hline $85 \mathrm{Rb}$ & 0,9992 & 0,37 & 0,41 & 2,77 & 5,46 & 1645 \\
\hline $88 \mathrm{Sr}$ & 0,9995 & 0,08 & 0,10 & 2,15 & 2,84 & 118 \\
\hline $96 \mathrm{Mo}$ & 0,9999 & 0,71 & 0,89 & 8,87 & 7,85 & 58,0 \\
\hline $105 \mathrm{Pd}$ & 0,9995 & 0,28 & 0,70 & 0,83 & 7,31 & 5,1 \\
\hline $111 \mathrm{Cd}$ & 0,9999 & 0,02 & 0,04 & 1,06 & 2,95 & 5,0 \\
\hline $118 \mathrm{Sn}$ & 0,9997 & 0,04 & 0,06 & 0,80 & 1,96 & 50,0 \\
\hline $122 \mathrm{Sb}$ & 0,9999 & 0,01 & 0,02 & 0,74 & 3,13 & 100 \\
\hline $125 \mathrm{Te}$ & 0,9999 & 0,07 & 0,15 & 1,43 & 7,02 & 25 \\
\hline $137 \mathrm{Ba}$ & 0,9999 & 0,09 & 0,13 & 1,54 & 7,72 & 42,5 \\
\hline $182 \mathrm{~W}$ & 0,9999 & 0,19 & 0,22 & 1,90 & 3,77 & 5,1 \\
\hline $195 \mathrm{Pt}$ & 0,9999 & 0,02 & 0,04 & 2,89 & 5,75 & 5,0 \\
\hline $202 \mathrm{Hg}$ & 0,9996 & 1,27 & 1,93 & 5,26 & 5,44 & 48,0 \\
\hline $205 \mathrm{TI}$ & 0,9997 & 0,45 & 0,77 & 4,91 & 3,45 & 10,0 \\
\hline $208 \mathrm{~Pb}$ & 0,9999 & 0,19 & 0,21 & 4,71 & 3,39 & 85,0 \\
\hline $209 \mathrm{Bi}$ & 0,9997 & 0,003 & 0,005 & 4,07 & 4,36 & 25,0 \\
\hline $238 \mathrm{U}$ & 0,9994 & 0,001 & 0,002 & 5,41 & 5,58 & 4,9 \\
\hline
\end{tabular}

$\mathrm{r}=$ coefficient de corrélation, $\mathrm{LD}=$ limite de détection, $\mathrm{LQ}=$ limite de quantification, Répét. = répétabilité,

Repro $=$ reproductibilité, Conc.$=$ niveau de concentration ayant servi aux mesures de répétabilité et de reproductibilité.

obtenues sont du même ordre voire inférieures à celles atteintes par la technique de spectrophotométrie d'absorption atomique électrothermique, les résultats étant exprimés dans les tableaux III, IV, V sur la dilution finale analysée (ils tiennent compte de la dilution de

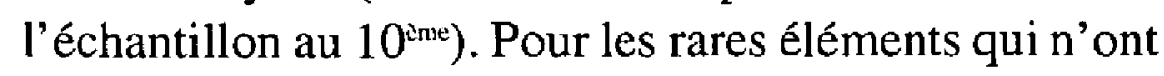
pas été validés, dans cette approchẹ multi-élémentaire la mise en œuvre soit de la DRC, soit d'une technique spécifique avec un mode opératoire particulier comprenant à la fois l'emploi d'un diluant et de conditions de mesure adaptées s'avèrent indispensables. Pour les éléments validés, s'agissant d'une approche de quantification multi-élémentaire, les conditions que nous avons retenues pour ces déterminations sont des compromis. L'emploi de méthodes optimisées pour chaque métal permet d'améliorer de manière notable les perfor- 
mances de l'ICP-MS comme le montrent Labat et coll. dans ce numéro (13). Il s'agit cependant d'une technique bien adaptée tant à la toxicologie clinique qu'à la toxicologie médicolégale, mais également à la surveillance d'un certain nombre d'éléments en médecine du travail (14) ou dans le cadre des effets des métaux lourds sur l'environnement et la santé (15).

\section{Conclusion}

Le dosage multi-élémentaire simultané d'un grand nombre d'éléments -environ 30- à partir d'un échantillon biologique limité constitue une approche nouvelle en toxicologie analytique. La technique, validée pour le plasma, le sang total et les urines, peut être mise en œuvre dans tous les laboratoires disposant d'une torche à plasma couplée à un spectromètre de masse. Elle est spécifique, sensible et reproductible pour l'ensemble des éléments étudiés. Elle présente l'avantage d'être à la fois plus rapide et plus sensible voire beaucoup plus sensible -à l'exception de l'aluminium- que la spectrophotométrie d'absorption atomique tout en permettant une quantification multi-élémentaire. Certes les performances peuvent être encore améliorées grâce à l'emploi de protocoles spécifiques parfaitement adaptés à un métal donné (13). L'ICP-MS est par ailleurs la seule technique permettant de doser certains éléments. Enfin la diminution de la taille et du coût de ce type d'appareil, constituent un apport déterminant dans le domaine de l'analyse toxicologique offrant ainsi des perspectives nouvelles pour une meilleure prise en charge des malades, tant dans le domaine de la toxicologie clinique, que de la surveillance professionnelle ou environnementale.

Cet équipement a été acquis grâce à un financement de l'Association pour la Fondation Charles Nicolle Rouen - France (Président Professeur Eric MALLET).

\section{Références}

1. Patriarca M. The contribution of inductively coupled plasma mass spectrometry to biomedical research. Microchem. J. 1996 ; 54 : 262-71.

2. Goullé J.P., Lacroix C. L'analyse toxicologique à l'aube du $3^{\text {ème }}$ millénaire. Ann. Biol. Clin. $2001 ; 59: 605-12$.

3. Chappuis P. Techniques d'analyse des oligo-éléments chez l'homme. Ed. TECH et DOC, Editions Médicales Internationales. 1995.

4. Pineau A., Guillard O. Techniques d'analyse des oligoéléments chez l'homme «volume $2 »$, Ed. TECH et DOC, Editions Médicales Internationales. 2001.
5. Taylor A., Branch S. Halls D.J., Owen L.M.W., White M. Atomic spectrometry update-clinical and biological materials, food and beverages. J. Anal. At. Spectrom. $1997 ; 12: 119 \mathrm{R}-221 \mathrm{R}$.

6. Taylor A., Branch S., Halls D.J., Owen L.M.W., White M. Atomic spectrometry update-clinical and biological materials, food and beverages. J. Anal. At. Spectrom. 1998 ; 13 : 57R-106R.

7. Taylor A., Branch S., Halls D.J., Owen L.M.W., White M. Atomic spectrometry update-clinical and biological materials, food and beverages. J. Anal. At. Spectrom. $1999 ; 14: 717-81$.

8. Taylor A., Branch S., Halls D.J., Owen L.M.W., White M. Atomic spectrometry update-clinical and biological materials, food and beverages. J. Anal. At. Spectrom. $2000 ; 15: 451-87$.

9. Taylor A., Branch S., Halls D.J., Owen L.M.W., White M. Atomic spectrometry update-clinical and biological materials, food and beverages. J. Anal. At. Spectrom. $2001 ; 16: 421-46$.

10. Taylor A., Branch S., Halls D.J., Patriarca M., White M. Atomic spectrometry update-clinical and biological materials, food and beverages. J. Anal. At. Spectrom. 2002 ; 17 : 414-55.

11. Taylor A., Branch S., Halls D.J., Patriarca M., White M. Atomic spectrometry update-clinical and biological materials, food and beverages. J. Anal. At. Spectrom. $2003 ; 18: 385-427$.

12. Protocole de validation de techniques. Commission validation de techniques de la Société Française de Biologie Clinique. L'information Scientifique du Biologiste. $1985 ; 11: 5-51$.

13. Labat L., Dehon B., Dhorne C., Lhermitte M. Dosage de métaux par ICP-MS dans différents milieux biologiques. Ann. Toxicol. Anal. 2003 ; 15 : 281-6.

14. Dehon B., Nisse C., Lhermitte M., Haguenoer J.M. Métaux et médecine du travail. Ann. Toxicol. Anal. $2001 ; 13: 203-19$.

15. Miquel G. Les effets des métaux lourds sur l'environnement et la santé. In : Rapport de l'office parlementaire d'évaluation des choix scientifiques et technologiques. 2001 avril, $n^{\circ} 27979$, Assemblée Nationale/n²61 Sénat. 